\title{
Los Institutos Politécnicos de Educación Superior (IPES) como una alternativa a la creación de nuevas universidades en la década de $\mathbf{1 9 7 0}$ en la Argentina
}

\author{
Higher Education Polytechnic Institutes as an \\ Alternative to the Creation of New Universities \\ in the 1970s in Argentina
}

\author{
Mariana Mendonça* \\ Universidad de Buenos Aires-CONICET \\ ORCID ID: 0000-0001-8125-6371
}

$\begin{array}{ll}\text { Recibido: } & 04 / 03 / 2020 \\ \text { Aceptado: } & 29 / 09 / 2020\end{array}$

DOI: https://doi.org/10.20318/cian.2020.5796

Resumen: La educación postsecundaria ha pasado a ser uno de los temas más importantes de la agenda política a nivel mundial desde el inicio de la segunda posguerra. El aumento de la demanda en dicho nivel generó un crecimiento institucional que dio lugar, a su vez, a un proceso de diversificación y diferenciación que se ha intensificado a lo largo de las últimas décadas, y ha adoptado formas distintas en cada país. Universidades
Abstract: Post-secondary education has become one of the most important issues on the global political agenda since the beginning of the Second World War. The increase in demand at this level generated institutional growth that in turn led to a process of diversification and differentiation that has intensified over the last few decades, and has taken different forms in each country. Public and private universities and non-university institutions,

"mmendonca85@gmail.com 
públicas y privadas e instituciones no universitarias, son sólo algunos de los numerosos tipos de unidades académicas que existen a lo largo y ancho de todo el mundo.

La Argentina constituye un caso emblemático de dicho proceso, dado que la expansión matricular e institucional se llevó a cabo, principalmente, en el sector universitario público. Pese a ello, existieron distintos proyectos que se presentaron desde mediados del siglo XX, en los que se proponía la implementación de modelos similares a aquéllos que funcionaban en otros países. En este trabajo nos analizaremos el proyecto del rector de la Universidad Nacional de Cuyo, presentado en 1972, y en el que se sugería la implementación de Institutitos Politécnicos de Educación Superior. Se trata de una propuesta relevante ya que, a pesar de que la expansión del sistema universitario acabó por cobrar una forma completamente distinta, su análisis permite arrojar luz en torno a los debates propios de la época respecto de qué forma concreta darle a dicho proceso expansivo.

Palabras clave: universidad, política, educación superior, diversificación, diferenciación. are just some of the many types of academic units that exist throughout the world.

Argentina is an emblematic case of this process, since the enrolment and institutional expansion took place mainly in the public university sector. In spite of this, there were different projects that were presented since the mid-twentieth century, which proposed the implementation of models similar to those in other countries. In this work we aim to analyze the proposal of the rector of the National University of Cuyo, formulated in 1972, and which suggested the implementation of Higher Education Polytechnic Institutions. This is a relevant project since, despite the fact that the expansion of the university system ended up taking on a completely different form, its analysis allows us to shed light on the debates that gave form to this expansion process.

Keywords: university, policies, higher education, diversification, differentiation

\section{Introducción ${ }^{1}$}

Los años de la segunda posguerra fueron un punto de inflexión en la educación superior a nivel mundial. El proceso de masificación que experimentaron las instituciones en todo el mundo fue clave en la formulación y lineamiento de nuevas políticas educativas. El paso de una universidad de elites a una de "masas" ${ }^{2}$ desencadenó nuevos problemas, no sólo vinculados con la organización de las casas de estudio, sino también con el perfil de los estudiantes que ahora ingresaban al nivel superior. El proceso de masificación habilitó el ingreso de sectores sociales antes excluidos de este nivel educativo, lo que obligó a repensar opciones y alternativas a los modelos tradicionales de educación superior.

\footnotetext{
${ }^{1}$ Agradecemos los comentarios y sugerencias de los/as revisores, los cuales han resultado en una mejora del trabajo aquí presentado.

${ }^{2}$ Martin Trow, Problems in the transition from elite to mass higher education (California: Institute of International Studies, University of California, 1973).
} 
Estados Unidos (EE.UU.) fue el primer país en experimentar un aumento matricular en el nivel postsecundario. Ya a fines de la década de 1960, el $40 \%$ del grupo de edad de entre 18 y 22 años se inscribía para cursar estudios superiores. Poco tiempo después, el sistema de educación superior de Canadá atravesó su propio proceso de masificación, mientras que los de Europa Occidental lo hicieron en la década de $1980^{3}$. Este panorama generó, asimismo, una especial atención en las investigaciones vinculadas con el sector. La masificación y el ingreso de grupos sociales antes excluidos trajeron aparejada la implementación de distintas medidas. En primer lugar, cabe señalar los circuitos diferenciados en la formación profesional: en EE.UU., se conformaron programas de dos años en los Colleges y de 4 años en las universidades, las cuales ofrecen, a su vez, la posibilidad de acceder a un Minor en dos años y/o un Major en cuatro años. Asimismo, se produjo una enorme proliferación de investigaciones que abordaron las problemáticas del abandono en el primer año de estudios, muy vinculado con los nuevos sectores sociales que ahora accedían a la educación superior.

En América Latina, el proceso de masificación también tuvo lugar a partir de mediados del siglo XX, aunque a una escala menor que los países mencionados. La tasa bruta de matrícula pasó de un 6\% en 1970 a un $17 \%$ en 1990, 23\% en el 2000 y alcanzó el $32.4 \%$ en el año 2006. En la Argentina, específicamente, esta masificación fue acompañada de una expansión institucional pública y privada. Sin embargo, los circuitos de diferenciación no lograron plasmarse, en términos formales, más allá de la división entre instituciones públicas y privadas, y universitarias y no universitarias. En efecto, no hubo trayectos diferenciados en la cantidad de años de cursada, como sí ocurrió, por ejemplo, en EE. UU. Por el contrario, entre 1971 y 1973 se crearon 16 nuevas universidades nacionales, pese a haberse presentado distintos proyectos alternativos para hacer frente al aumento de la demanda en el sistema de educación superior. Con ello se dio inicio a la primera ola de expansión universitaria. La segunda tuvo lugar en la década de 1990, y la tercera se inició en los últimos años de la década del 2000. Estos distintos ciclos, asimismo, fueron acompañados por la creación de instituciones privadas, e instituciones no universitarias.

Sin embargo, este proceso no dio lugar a una diferenciación clara en los trayectos de formación. Las funciones, misiones y objetivos de todas estas instituciones, de hecho, tienden a superponerse. Así, por ejemplo, tanto

\footnotetext{
${ }^{3}$ Ana María Ezcurra, Igualdad en educación superior. Un desafío mundial (Buenos Aires: Universidad Nacional de General Sarmiento, 2011).
} 
los institutos terciarios de formación docente como las universidades nacionales expiden títulos de profesores: si bien estas últimas también expiden títulos de Licenciados e Ingenieros, es posible realizar el trayecto pedagógico al igual que los profesorados. Asimismo, a lo largo de las distintas olas de expansión, las universidades nacionales han ampliado su oferta y también expiden títulos técnicos, superponiéndose con las carreras ofrecidos por las instituciones no universitarias.

La creación de nuevas universidades nacionales desde mediados de la década del cincuenta hasta el año 2015 se ha analizado como una política que adoptó una forma anárquica ${ }^{4}$. Asimismo, al analizar la educación postsecundaria argentina en su conjunto, es posible observar una situación similar, principalmente por la superposición de títulos, objetivos y funciones de todas las instituciones. En el año 2017 había un total de 13872 títulos ofrecidos en todo el territorio nacional por 2383 instituciones formadoras. De ellas, el 65\% pertenecía a un régimen estatal (público) y el 35\% restante al sector privado 5 .

A lo largo de este proceso de expansión matricular e institucional, sin embargo, hubo distintas propuestas y proyectos presentados. Especialmente en el período de posguerra, y hasta mediados de la década de 1980, la educación estuvo muy vinculada con el planeamiento. Distintas agencias internacionales (USAID, Ford, Rockefeller) asesoraron a los gobiernos en la planificación educativa, especialmente en el nivel superior ${ }^{6}$. La idea de que el desarrollo de la ciencia y la tecnología permitirían un crecimiento económico, fue clave en las decisiones que se tomaron en aquellos años. Consecuentemente, el eje estuvo puesto en la necesidad de transformar los modelos de educación superior hasta ese entonces existentes. En toda la región el modelo predominante era el napoleónico, de origen francés y la propuesta en estos años era asemejarlo al sistema anglosajón. Rudolf Atcon fue una

${ }^{4}$ Adriana Chiroleu, Claudio Suasnábar y Laura Inés Rovelli, Política universitaria en la Argentina: revisando viejos legados en busca de nuevos horizontes (Buenos Aires, Universidad Nacional de General Sarmiento, 2012).

${ }^{5}$ Danya Tavela y Magalí Catino, Áreas de vacancia, vinculación, pertinencia y planificación del sistema universitario: una herramienta para abordar la expansión de la educación superior en territorio (Buenos Aires, Secretaría de Políticas Universitarias-Ministerio de Educación, 2018).

${ }^{6}$ Pedro Krotsch, Educación Superior y reformas comparadas, (Quilmes: Universidad Nacional de Quilmes, 2009): 154. Para un análisis más detallado de la influencia de los organismos internacionales, véase Adriana Chiroleu, "La modernización universitaria en la agenda de gobierno argentino: lecciones de la experiencia", en Fundamentos en Humanidades, v. V, n. 9 (2004). 
figura clave en este sentido, ya que asesoró a los distintos gobiernos de la región ${ }^{7}$. En la Argentina, en particular, fueron años de fuertes debates y discusiones en torno al rumbo que debía tomar la universidad. Esta institución con una fuerte tradición en el país, ya había atravesado los primeros cambios a mediados de los años cincuenta con la expansión matricular, la creación de universidades privadas tras la sanción del decreto-ley de 1955 y la creación de las primeras casas de estudio con un perfil regional. Sin embargo, a fines de los años sesenta fueron publicados distintos diagnósticos e informes que daban cuenta de los problemas que presentaba la educación superior en el territorio nacional. Paralelamente, surgieron distintas propuestas y alternativas para poder brindar posibles respuestas. Algunas generaron discusiones al interior de los organismos públicos mientras que otros, en cambio, pasaron casi desapercibidos ${ }^{8}$. En la mayoría es posible encontrar similitudes con los modelos extranjeros, principalmente los implementados en EE. UU.

En este trabajo, nuestra intención es indagar en uno de los proyectos presentado a principios de la década de 1970 , y que tuvo muy poca repercusión. Argumentaremos que el mismo proponía una alternativa, con un trayecto de formación diferenciada, para dar respuesta a las altas tasas de inscripción en las universidades nacionales. En este sentido, los Institutos Politécnicos de Educación Superior (IPES) se propusieron como una alternativa no sólo frente a la alta tasa de matrícula, sino también para dar respuesta a los problemas vinculados con la elección mayoritaria de carreras tradicionales, la alta tasa de abandono en el primer año y la extensión en los años de cursada y la baja tasa de graduación. Se trata de un proyecto relevante ya que, a pesar de que la expansión del sistema universitario acabó por cobrar una forma completamente distinta, su análisis permite arrojar luz en torno a los debates propios de la época respecto de qué forma concreta darle a dicho proceso expansivo. Asimismo, permite repensar el devenir del sistema de educación superior en la Argentina, que sigue siendo eje de debate en la agenda política y académica.

${ }^{7}$ Krotch, Educación Superior. Para un análisis del caso de Uruguay, véase María Eugenia Jung, "Derechas y universidad en los sesenta. Lecturas inspiradoras y modelos universitarios: Tres estudios de caso en Uruguay y Argentina", en Cuadernos de Marte, n. 17 (2019).

${ }^{8}$ Mariana Mendonça, "Cómo Resolver El Problema Universitario: Nuevos Diagnósticos Y Cambios En La Agenda Política Durante El Onganiato (1966-1970)", Historia da Educaçao Volumen 19 (2015): 229-248 y Mariana Mendonça, "Creación, nacionalización y escisión: ¿reestructuración? Una aproximación al proceso de transformación del sistema universitario argentino (1971-1973)", Revista Iberoamericana de Educación Superior Volumen 9, número 24 (2018): 82-105. 
Para avanzar con este análisis, nos valdremos de las actas del Consejo de Rectores de Universidades Nacionales, como así también la revista editada por dicho organismo. Argumentaremos, de manera general, que éstos Institutos se asemejaban a los modelos de educación superior técnica que comenzaron a ofrecerse en otros países del mundo como respuesta al proceso de masificación. Sin embargo, la diferenciación en los trayectos de formación en la Argentina acabó tomando una forma completamente distinta. En el marco de la tercera ola de expansión universitaria, este trabajo se propone repensar el devenir del sistema universitario en el país, y así echar luz sobre los trayectos de formación y los procesos de diferenciación.

De este modo, en el primer apartado daremos cuenta de la expansión y diferenciación que se llevó a cabo en el sistema de educación postsecundario a nivel mundial en el período de posguerra. Luego analizaremos el caso de la Argentina, en donde la respuesta a la expansión matricular fue la creación de nuevas universidades nacionales. En el tercer apartado, daremos cuenta de un proyecto alternativo a la forma que finalmente adoptó el proceso de expansión universitaria, presentado por el rector de la Universidad Nacional de Cuyo. Finalmente, nos proponemos mostrar las similitudes que tuvo éste proyecto con modelos extranjeros.

\section{Expansión y diferenciación en la posguerra}

Desde el inicio del período de posguerra hasta mediados del siglo XX, la matrícula de educación superior aumentó a un ritmo considerable a nivel mundial. Distintos países, sin importar su grado de industrialización, afrontaron el aumento de la demanda en dicho nivel. Si bien en un primer momento la expansión institucional estuvo directamente vinculada con el desarrollo económico, posteriormente fue asociada con los procesos de democratización de cada espacio nacional. Como señala Trow ${ }^{9}$, el crecimiento en la matrícula fue acompañado por transformaciones en las estructuras universitarias. Por un lado, debido a cuestiones vinculadas con sus tradiciones, funciones y presupuesto, las universidades de elite no pudieron absorber el aumento matricular. Y, por el otro, parte de estas instituciones debieron transformarse en universidades de masas, incorporando una gran cantidad de funciones nuevas. Este proceso de reestructuración, señala el autor, resultó en una fuerte diferenciación no sólo en el perfil de los ingresantes, lo que se evidencia

\footnotetext{
${ }^{9}$ Trow, Problems in the transition from elite to mass higher education.
} 
en aspectos como la edad de ingreso o el currículo, sino también en su trayectoria académica y el tipo de formación recibida al ingresar al sistema de educación superior, existiendo diferencias en la calidad de dicha formación, como así también en la forma que cobraba la interacción entre estudiantes y docentes. Incluso las propias instituciones comenzaron a diferenciarse entre sí en cuanto al tamaño, la administración y las formas de gobierno. En efecto, junto con el proceso de masificación, se inauguró una primera fase de diversificación institucional mediante la formación de trayectos educativos diferenciados. Cada país adoptó formas distintas: algunos establecieron un sistema universitario público y privado, mientras que otros optaron por mantener el sistema universitario público para un grupo reducido de estudiantes y crear nuevas instituciones no universitarias para un público masivo. Otros países, en cambio, iniciaron un proceso de masificación de las universidades públicas y dejaron la educación "de elite" para las de gestión privada. En definitiva, la expansión institucional resultó en una variada oferta de educación postsecundaria, que incluyó universidades tradicionales orientadas a la investigación y otras orientadas a la formación profesional, así como institutos de formación profesional, de formación docente, y de formación técnica.

En EE. UU., por ejemplo, se crearon los Community Colleges con el objetivo de dar respuesta al aumento de la demanda en el nivel superior. Estas instituciones ofrecen carreras de 1 o 2 años de duración, con orientación técnica y/o profesional. Asimismo, aquellos estudiantes que decidan continuar sus estudios en un programa de formación de 4 años pueden hacerlo. Eso es posible debido al formato que presentan los estudios de 4 años de las universidades, las cuales están conformadas por una formación básica y general de 2 años, en el cual se obtiene el minor y luego se continúa la formación especializada para obtener el major. Aquellos estudiantes que acceden al título después de culminar un programa de 4 años, pueden optar por realizar un master o un doctorado. En definitiva, hay una oferta académica y profesional diversa y diferenciada. Esto, a su vez, genera una diferenciación en la formación de la fuerza de trabajo. En palabras de Altbach, et. al., esta diferenciación en los trayectos de formación tiene un fuerte impacto en la estratificación socioeconómica, pudiendo mejorarla o empeorarla ${ }^{10}$.

Alemania y Francia son otros ejemplos de marcada diferenciación en los trayectos de formación profesional. Si bien en ambos casos, al igual que en EE. UU., es posible navegar entre los distintos trayectos educativos, hay

\footnotetext{
${ }^{10}$ Altbach, Philip G., Liz Reisberg, and Hans de Wit, eds. Responding to massification: Differentiation in postsecondary education worldwide (Springer, 2017).
} 
una marcada diferencia entre las formaciones técnicas, profesionales y la investigación. En Alemania, el sistema de educación superior está integrado por tres tipos de instituciones: las Universitäten (universidades), las Fachhochschulen (escuelas superiores especializadas) y las Kunst und Musikschulen (escuelas de arte y música). Es sobre las primeras que se consolidó el modelo humboldtiano de educación superior, ya que están orientadas principalmente a la investigación. En efecto, sólo estas instituciones pueden conferir títulos de doctorado. Por otra parte, si bien algunas se especializan por disciplinas, otras abarcan la generalidad de las ramas de estudio. Las segundas, en cambio, fueron creadas a partir de la década de 1970 en el marco de la masificación de la matrícula, y se caracterizan por tener un perfil más profesional y práctico. Las carreras que ofrecen son aquellas vinculadas con las ingenierías, economía, administración de empresas, trabajo social y diseño, entre otras. Si bien se realiza investigación, se halla orientada a la práctica y focalizada en la orientación ocupacional de sus egresados. Estas casas de estudio, por otra parte, no suelen expedir títulos de doctor. El tercer tipo de institución, tal como su nombre lo indica, está orientada a la formación, en bellas artes, teatro, y música, tanto artística como en las áreas de dirección, producción y guion. Asimismo, ofrecen carreras de las áreas de diseño, arquitectura y medios de comunicación. Todas las instituciones, sean éstas públicas o privadas, están sujetas a la legislación de la educación superior tanto para la elaboración de los programas de estudio como para la concesión de títulos. Las instituciones públicas, asimismo, reciben financiamiento del gobierno estatal y federal. Existen también universidades religiosas y privadas. Éstas últimas, en la mayoría de los casos, ofrecen carreras vinculadas con las ciencias aplicadas. En Francia, por su parte, se puede optar por ingresar a las universidades, a los Instititut Universitaires de Tecnologie (IUT), a las Section de Technicien Superieur (STS), o a las Grandes Ecoles. La educación superior no universitaria, por su parte, está conformado mayormente por carreras cortas técnicoprofesionales que se dictan en los IUT y en los STS. El primer ciclo de estos estudios dura 2 años, y si bien es posible continuar con una formación de Maestría y Doctorado, son pocos los estudiantes que realizan esta trayectoria. La mayoría de quienes ingresan a estas instituciones, suelen haber aprobado el Bac Profesional, que los habilita a vender su fuerza de trabajo con un título de educación superior no universitario. Cabe señalar que la estructura del sistema de educación superior francés está caracterizada por una aceitada articulación entre sus ciclos. Todos los diplomas del primer ciclo equivalen a la Licenciatura; el título de doctor se obtiene luego de tres a cinco años de estudio más la realización de una tesis; la Habilitation se obtiene luego de 
transcurridos entre cuatro y diez años del doctorado, sobre la base de la contribución global del investigador como académico ${ }^{11}$.

En suma, a partir de la descripción que acabamos de realizar, es posible señalar algunas características de las transformaciones implementadas durante la segunda mitad del siglo XX en el sistema de educación superior. A medida que la matrícula se fue incrementando, estos países fueron adoptando distintas acciones para absorber la demanda, principalmente diversificando la oferta. En Europa se desarrollaron dos estrategias para brindar carreras cortas de educación superior: la creación de establecimientos no universitarios de educación superior, por un lado; y la diversificación de la oferta académica dentro de las mismas universidades mediante la introducción de carreras cortas y/o con orientación profesional, por el otro. En EE.UU., asimismo, se optó por la diversificación institucional y la adopción de un sistema binario de instituciones universitarias y no universitarias ${ }^{12}$. Incluso al interior de los ciclos más largos de formación, nos encontramos con caminos diferenciados dependiendo de la carrera que se elija, sea ésta "profesional" o de "investigación".

Por su parte, hubo distintas respuestas al aumento de la demanda en los países latinoamericanos. Según Krotch, aquellos sistemas más jóvenes fueron más permeables para la introducción de nuevos modelos ${ }^{13}$. En México, Uruguay y Venezuela, por ejemplo, las universidades públicas se expandieron y diversificaron y también se crearon nuevas instituciones no universitarias de gestión pública a nivel regional con el objetivo de absorber la demanda. En Brasil, Chile y Colombia, en cambio, la educación superior de gestión pública se mantuvo restringida y fueron las de gestión privada las que dieron respuesta al aumento de la matrícula ${ }^{14}$.En este sentido, de acuerdo a García de Fanelli y Balán ${ }^{15}$ estas experiencias han contrastado con la europea. En primer lugar, se intentó absorber dicha demanda con una mayor oferta de instituciones tradicionales y carreras profesionales. En se-

${ }^{11}$ Osvaldo Barsky y Mabel Dávila, "Las carreras de posgrado en la Argentina”, en Los desafíos de la Universidad Argentina, ed. Osvaldo Barsky, Víctor Sigal y Mabel Dávila (Buenos Aires, Universidad de Belgrano, Siglo XXI editores Argentina, 2004): 42.

${ }^{12}$ Ana María García de Fanelli y Augusto Trombetta, Diferenciación institucional y reformas curriculares en los sistemas de educación superior (Buenos Aires, 1996).

${ }^{13}$ Krotch, Education Superior: 158.

${ }^{14}$ Thorn Holm-Nielsen, Joaquín Brunner y Jorge Balán, 2005, pág. 41) “Regional and international challenges to higher education in Latin America", en Higher Education in Latin America. The international dimension, ed. Hans de Wit, Isabel Jaramillo, Jocelyne Gacel-Ávila y Jane Knight (Washington D. C.: The World Bank, 2005):41.

${ }^{15}$ Ana María García de Fanelli y Jorge Balán, Expansión de la oferta universitaria: nuevas instituciones, nuevos programas (Buenos Aires, 1994). 
gundo lugar, la creación de instituciones no universitarias no ha significado un cambio en el perfil de las universidades tradicionales, ya que muchas de ellas ofrecen también carreras profesionales y de larga duración. Por último, la expansión institucional no tuvo lugar únicamente en el sector público, sino que también ocupó un lugar importante la creación de instituciones de educación superior de gestión privada. Con la excepción de Cuba, es posible encontrar una amplia oferta de gestión privada en toda la región ${ }^{16}$. Krotch sostiene que el fuerte desarrollo de este sector debe analizarse como una consecuencia ante la ausencia de opciones de formación como las que se implementaron en Europa y EE. UU., esto es, los trayectos de formación con una opción vocacional tecnológica y la de los Community Colleges ${ }^{17}$.

El caso de la Argentina es bastante particular, ya que pese a haber atravesado fuertes trasformaciones en el sistema de educación superior, en estas décadas se llevó a cabo una política de expansión institucional en el sector universitario. Si bien, tal como hemos mencionado, se diversificó el sistema con la creación de universidades privadas, el foco estuvo localizado en el sector universitario público. En el marco de dicha política se intentó incorporar modelos académicos nuevos, con reformas basadas principalmente en los países anglosajones. Sin embargo, dichas reformas no prosperaron y los problemas vinculados con el aumento de la matrícula, la alta tasa de abandono y la baja tasa de egresados se mantuvieron. En este contexto, surgieron otras propuestas como alternativas a implementarse en el sistema de educación superior. A continuación, nos proponemos analizar una de ellas, presentada por el Rector de la Universidad Nacional de Cuyo en 1972. La particularidad de la misma radica en su similitud con aquellas propuestas implementadas en otros países y, a su vez, la poca -sino nula- trascendencia que tuvo en aquellos años. Para avanzar sobre esta cuestión, comenzaremos por contextualizar brevemente el proceso de expansión matricular e institucional.

\section{La expansión matricular e institucional en la Argentina de los años sesenta}

A partir de la década de 1950, el sistema universitario argentino atravesó un fuerte proceso de masificación. Las dos nuevas leyes universitarias sancionadas por el gobierno de Perón en 1947 y 1954 postulaban la integración de la universidad a los objetivos de justicia social y soberanía nacional. Entre las

\footnotetext{
${ }^{16}$ Holm-Nielsen, Brunner y Balán, "Regional and international challenges to higher education in Latin America".

${ }^{17}$ Krotch, Educación Superior: 158.
} 
medidas que destacan, cabe mencionar aquéllas que impulsaron el aumento de la matrícula compuesta por una fuerte presencia de los sectores de bajos recursos. En 1950 se eliminaron los aranceles, garantizando la gratuidad del sistema educativo superior por primera vez en la historia, se eliminaron los exámenes de ingreso, y se implementó una serie de becas para los estudiantes de familias obreras. De este modo, mientras que por cada mil habitantes había tres estudiantes universitarios en 1945, el promedio trepó a ocho en 1955 llegando casi a triplicarse en apenas diez años. Tal como ya hemos mencionado, esta política formó parte de una tendencia mundial ${ }^{18}$.

Al igual que en otros países, el proceso de masificación trajo aparejados problemas vinculados con la baja tasa de egresados, el aumento del abandono, la extensión en la cursada por sobre el tiempo teórico estimado, entre otros. Asimismo, en 1949 comenzaron los problemas presupuestarios, situación que repercutió en la infraestructura de las casas de estudio, que no estaban preparadas para recibir a semejante población estudiantil. Si bien se formularon proyectos de mejora y expansión de los edificios, quedaron inconclusos ${ }^{19}$.

El golpe de Estado de 1955 puso fin al gobierno de Perón, y las casas de estudio comenzaron a transitar una etapa de "desperonización": las universidades fueron intervenidas por el gobierno de facto y los grupos estudiantiles que se habían opuesto al régimen del gobierno anterior ocuparon las casas de estudio, impidiendo el ingreso a las autoridades peronistas ${ }^{20}$. En este marco, y frente a lo que se consideró como un crecimiento excesivo de la población universitaria, el nuevo gobierno decretó la creación de universidades privadas. Dicha ley generó una fuerte oposición, pero finalmente se dio luz verde a las instituciones de gestión privada, inauguró la primera fase de diversificación de la oferta de educación superior, ya que hasta este entonces las 8 universidades eran de gestión pública. Paralelamente, se crearon dos nuevas universidades nacionales sobre la base de institutos ya existentes, la Universidad del Nordeste en Corrientes y la Universidad del Sur en Bahía Blanca. También se rebautizó la Universidad Obrera bajo el nombre de Universidad Tecnológica Nacional ${ }^{2122}$.

${ }^{18}$ Pablo Buchbinder, Historia de las universidades argentinas (Argentina: Editorial Sudamericana, 2005).

${ }^{19}$ Buchbinder, Historia de las universidades argentinas: 160.

${ }^{20}$ Buchbinder, Historia de la universidad argentina: 169.

${ }^{21}$ Daniel Cano, La educación superior en la Argentina (Buenos Aires, Grupo editor latinoamericano, 1985): 14.

${ }^{22}$ Bajo el gobierno de Perón se había creado la Universidad Obrera, con el objetivo de formar "Ingenieros de fábricas". 
Estas medidas formaron parte de un proceso mayor de modernización de las casas de estudio en el país. Surgieron nuevas carreras como sociología y psicología en 1957, y se reformaron los planes de estudio. Asimismo, la carrera de ciencias de la educación reemplazó a pedagogía. Un año después se creó la carrera de ciencias antropológicas y la de economía. También se intentó reorientar la matrícula, logrando un aumento de alumnos en la Facultad de filosofía y letras tras la apertura de psicología y un $60 \%$ más de inscriptos en las carreras de ciencias exactas. Con el mismo objetivo se creó el Departamento de Orientación Vocacional, ya que se consideraba la falta de este como uno de los factores más influyentes en la deserción. En el marco de este proceso de modernización, se comenzó a construir el primer pabellón de Ciudad Universitaria en Buenos Aires ${ }^{23}$.

Sin embargo, y a pesar de las grandes transformaciones que se llevaron a cabo en este período, se debe tener en cuenta que este proceso no fue homogéneo, y tomó formas distintas en cada provincia, Facultad y carrera. Por otra parte, la renovación y modernización institucional fue perdiendo fuerza al comenzar la década de 1960. Tras los primeros pasos de normalización, comenzaron a brotar tensiones entre los miembros de la comunidad, quienes tenían diferencias en la orientación académica y científica. Asimismo, las diferencias políticas existentes se profundizaron tras la Revolución Cubana, y generaron la radicalización política de una parte importante de los estudiantes ${ }^{24}$.

Las políticas llevadas adelante por el gobierno de la autodenominada Revolución Argentina, presidida inicialmente por el general Onganía, tuvieron un fuerte impacto en la vida universitaria. Como respuesta a los problemas que preocupaban al gobierno militar fueron llevadas a cabo una serie de políticas que podemos dividir esquemáticamente en dos etapas. En la primera de ellas se intentó descongestionar las universidades mediante un régimen represivo que implementó, además, medidas de ingreso selectivo. Estos intentos de normalización de las casas de estudio pronto se vieron frustrados, lo que llevó al gobierno de facto a encarar una segunda etapa: el redimensionamiento de las universidades. Esto generó fuertes debates en la agenda pública nacional: las discusiones, la elaboración de proyectos y propuestas incluyeron no sólo al Ministro de educación, sino también a los

${ }^{23}$ Buchbinder, Historia de las universidades argentinas y Chiroleu, "La modernización universitaria".

${ }^{24}$ Buchbinder, Historia de las universidades argentinas. 
rectores de las universidades nacionales, decanos de algunas Facultades, e incluso personalidades vinculadas con organismos extranjeros ${ }^{25}$.

\section{Nuevas universidades}

La posibilidad de crear nuevas universidades nacionales concitó tanto apoyos como rechazos hacia finales de la década de 1960. La campaña por la creación de nuevas universidades nacionales en distintas partes del territorio nacional fue encabezada por Alberto Taquini (h), decano de la Facultad de Farmacia y Bioquímica de la Universidad de Buenos Aires. En su trabajo titulado "Nuevas universidades para un nuevo país", da cuenta de algunas de las características que había asumido el sistema de educación superior universitario en la Argentina y, consecuentemente, propone la creación de cinco universidades nacionales. Dichos indicadores coincidían con los diagnósticos que habían sido elaborados por el Consejo Nacional de Desarrollo y por el Consejo de Rectores de Universidades Nacionales (CRUN). Sin embargo, la propuesta de éstos últimos difería de la de aquél. Sucede que los organismos nacionales entendían que el sistema universitario se encontraba atravesando problemas relativos a la masificación, pero también sostenían que la creación de nuevas universidades nacionales no necesariamente los solucionaría. Puntualmente, entre sus argumentos, afirmaban que existían casas de estudios de reciente creación que aún no había logrado alcanzar una matriculación acorde con la inversión allí realizada ${ }^{26}$. Finalmente, tras marchas y contramarchas, el gobierno de Alejandro Agustín Lanusse dispuso en 1971 la creación de cinco universidades nacionales en el interior del país, procurando que éstas se amoldaran a las ideas de la época ${ }^{27}$. Así, se buscó dar a las nuevas casas de estudio un perfil moderno y cientificista, diferenciándolas de las universidades tradicionales en su estructura interna, su tamaño, y la oferta de carreras orientadas a las necesidades económicas

${ }^{25}$ Mariana Mendonça, "Cómo resolver el problema universitario: nuevos diagnósticos y cambios en la agenda política durante el onganiato (1966-1970)", Revista Historia da educaçao, v. 19, n. 47 (2015).

${ }^{26}$ Se trataba de las universidades creadas en 1956, la Universidad Nacional del Sur y la Universidad Nacional del Nordeste y la Universidad Nacional de Rosario que había comenzado a funcionar en 1969. Para un análisis más detallado, véase Mariana Mendonça, "La creación de nuevas universidades nacionales en la década de los años setenta. Continuidades y rupturas del plan Taquini en el marco de la coyuntura política nacional (1966-1973)", Perfiles Educativos, v. 37 (2015b).

${ }^{27}$ Laura Rovelli, “Del plan a la política”, en Temas y Debates, v. 17 (2009). 
de la región. Se pretendía con ello formar recursos humanos de carácter técnico y científico, que pudieran insertarse en las estructuras productivas de las distintas áreas del país. Así, la primera nueva universidad fue creada en 1971 en el sur de la provincia de Córdoba, en la ciudad de Río Cuarto, a la que siguieron la Universidad Nacional del Comahue en la región patagónica, las de Salta y Santiago del Estero, y las de Lomas de Zamora y Luján en el Área Metropolitana de Buenos Aires. De este modo, hacia 1973 la Argentina contaba ya con 13 nuevas universidades nacionales ${ }^{28}$.

Mientras comenzaba a implementarse el proceso expansivo que tuvo por base una multiplicación de universidades nacionales sobre todo el territorio, emergía una propuesta paralela de otra naturaleza. Se trata de la creación de Institutos Politécnicos de Educación Superior (IPES), elaborada por el rector de la Universidad Nacional de Cuyo y presentada en una de las reuniones mensuales que realizaba el Consejo de Rectores de Universidades Nacionales. A continuación, describiremos aquella propuesta.

\section{Los IPES: Una política de expansión del nivel terciario}

En 1972 el proceso de creación de nuevas universidades nacionales finalmente se había puesto en marcha, y ya se habían inaugurado 2 de las 16 casas de altos estudios que se sumaron al sistema universitario público de la Argentina. Es en este contexto que emergió el proyecto de los Institutos Politécnicos de Educación Superior.

El proyecto de Herrera se presentó a discusión en una de las reuniones mensuales que realizaban los rectores. El CRUN estaba conformado por los rectores de las universidades nacionales y las reuniones que se realizaban una vez por mes tenían como propósito discutir problemáticas de las casas de estudios, elaborar proyectos y evaluar la materialización de los mismos. Ese mismo organismo había discutido y propuesto alternativas ante los diagnósticos negativos de la performance de las universidades nacionales a fines de la década de $1960^{29}$. Había un acuerdo generalizado por parte de los rectores de evitar la creación de nuevas universidades. En cambio, proponían la

${ }^{28}$ Para un análisis más detallado de éste proceso, véase Mariana Mendonça, "Creación, nacionalización y escisión: ¿reestructuración? Una aproximación al proceso de transformación del sistema universitario argentino (1971-1973)", Revista Iberoamericana de Educación Superior, v. 9, n. 24 (2018).

${ }^{29}$ Mendonça, "Cómo resolver el problema universitario". 
reestructuración de las instituciones ya existentes ${ }^{30}$. El argumento de mayor peso era el presupuestario, ya que muchas de las casas de estudio, tal como hemos señalado, presentaban problemas por la falta de financiamiento. Las universidades públicas en la Argentina reciben un presupuesto anual por parte del gobierno nacional. La creación de nuevas instituciones significaría, entre otras cosas, un reparto de dichos fondos. Así como Devoto había propuesto una reestructuración de la UBA, sus pares también proponían alternativas a la creación de nuevas casas de estudio. Sin embargo, esta última opción se llevó a cabo a medias, ya que la política de expansión avanzó rápidamente y en poco tiempo se sancionaron las leyes de creación ${ }^{31}$.

Pese a haber participado y aprobado finalmente -junto con otros rectores- algunos de los planes de factibilidad correspondiente a las nuevas universidades, Herrera insistió en más de una oportunidad en la necesidad de elaborar una política nacional universitaria que contemplara todos los problemas relativos a la educación superior, poniendo especial énfasis en la formación de técnicos. Así lo testimonian las actas del CRUN del 17 de abril de 1972, cuando el rector de la Universidad Nacional de Cuyo, en las discusiones relativas a la política de creación de nuevas universidades, afirmó que existía una necesidad imperiosa "de creación de estudios de nivel terciario en [el] país". Consecuentemente, en dicha reunión afirmó también que antes de continuar con la creación de nuevas universidades era necesario estudiar una política universitaria de forma coordinada ${ }^{32}$.

Tres meses después, en la reunión del 13 de julio, Herrera presentó su proyecto para la creación de los Institutos Politécnicos de Educación Superior. El objetivo era brindar una respuesta a la expansión de la matrícula universitaria, ya que según el rector, la creación de nuevas universidades nacionales no sólo no parecía ser capaz de encauzar adecuadamente dicha expansión, sino que incluso había agravado los problemas preexistentes por falta de planificación ${ }^{33}$. Da acuerdo a Herrera, la expansión de los últimos

${ }^{30}$ En 1968 Raúl Devoto, rector de la Universidad de Buenos Aires (UBA) fue quien elaboró una propuesta para reorganizar dicha casa de estudios, aunque finalmente no se llevó a cabo. Véase Mariana Mendonça, "Los (frustrados) intentos de modernización en la Universidad de Buenos Aires desde su creación hasta el rectorado de Devoto", en Universidad, política y movimiento estudiantil en la Argentina entre la "Revolución Libertadora" y la democracia del 83', ed. Mariano Millán (Buenos Aires: Final Abierto, 2014).

${ }^{31}$ Mendonça, "Creación, nacionalización y escisión".

${ }^{32}$ Consejo de Rectores de Universidades Nacionales, Actas del Consejo de Rectores de Universidades Nacionales (Buenos Aires, 1972).

${ }^{33}$ Consejo de Rectores de Universidades Nacionales, Actas del Consejo de rectores de Universidades Nacionales (Buenos Aires, 1972). 
años había sido mayor en la enseñanza no universitaria. Asimismo, afirmaba que las universidades estaban dedicadas fundamentalmente a carreras básicas y de posgrado. En el año 1971, de 1579 oportunidades de carreras que ofrecía la universidad nacional, 964 (61\%) estaban dedicadas a las carreras básicas o largas; 432 (27\%) a carreras de posgrado y sólo 183 (12\%) a carreras cortas. Esto daba cuenta, a su vez, de la escasa importancia relativa que se le otorgaba a las carreras cortas, sobre todo en las áreas de Ciencias Básicas y Tecnológicas. Los alumnos matriculados en las carreras cortas eran 6845, de los cuales 2419 (35.3\%) correspondían a Ciencias Médicas; 2202 (32.2\%) a Ciencias Sociales; 1190 (17.4\%) a Ciencias Básicas y Tecnológicas; y $1034(15.1 \%)$ a Humanidades. Por su parte, al considerar los institutos de educación superior no universitarios, daba cuenta de que la gran proporción de alumnos y egresados estaban dedicados fundamentalmente al área Humanidades, especialmente a la formación docente ${ }^{34}$.

Frente a esta situación, el rector de la UNCuyo partió de las siguientes ideas para elaborar la propuesta de los IPES. Por un lado, se propuso permitir el ingreso masivo al nivel no universitario, atendiendo al aumento sostenido de la matrícula en el nivel superior. Por otra parte, el proyecto intentó dar una respuesta nueva que implicara "un cambio en la educación y una educación para el cambio". En este sentido, enmarcó su propuesta dentro del diagnóstico general de educación superior, procurando diversificar las oportunidades educativas a través de carreras cortas no tradicionales y que tuvieran pertinencia y fueran asunto de interés nacional.

\section{Objetivos y características generales}

Los IPES, entonces, tendrían como objetivo fundamental la formación de técnicos en el nivel superior a través de carreras cortas. De esta forma, se pretendía atender a la demanda creciente de estudios superiores, diversificar significativamente la oferta educativa no universitaria y dar oportunidad a todas las regiones o zonas del país de contar con instituciones de dicho nivel , un reclamo que venía gestándose en varias provincias tras el anuncio de creación de universidades. Asimismo, se procuraba racionalizar los esfuerzos financieros que se hacían en el país en relación a la expansión de los estudios superiores.

\footnotetext{
${ }^{34}$ Julio Herrera, Una política de expansión del nivel terciario (Mendoza, Universidad Nacional de Cuyo, 1972). En esta publicación se presentó, de manera detallada, el proyecto presentado ante el CRUN.
} 
Los Institutos tendrían objetivos propios, vinculados a la finalidad profesional de las carreras y cursos que se ofrecerían, y buscarían capacitar a los alumnos para el ingreso al mundo del trabajo. Asimismo, el proyecto establecía que la formación técnica debía integrarse con una formación general que atendiera, a su vez, a las necesidades de desarrollo de la persona y a la comprensión y participación en el proceso de cambio. Para ello, proponía una organización con la flexibilidad suficiente que permitiera una rápida adecuación a la demanda, posibilitando el cambio de sus carreras y especialidades y ofreciendo cursos de actualización. Esto, a su vez, permitiría una articulación con los estudios universitarios, permitiendo un reconocimiento recíproco de estudios a través de equivalencias entre universidades e institutos.

Otra característica que destaca es que se proponía realizar investigación aplicada y ofrecer servicios de orientación profesional a los alumnos graduados. Por otra parte, se proyectaba una vinculación de los institutos con las empresas o servicios donde podrían insertarse laboralmente los futuros egresados. Para ello, se procuraría ubicarlos próximos a los complejos industriales o centros que demandasen técnicos superiores.

Para la absorción del número creciente de la matrícula se proponía un ingreso amplio, el cual se realizaría de forma directa, con el único requisito de poseer un título del nivel medio de cualquier modalidad. El currículo sería flexible, procurando la mayor movilidad del estudiante entre las posibilidades educativas y la atención de las inquietudes individuales. Asimismo, se establecería una adecuada regulación legal para asegurar el ámbito ocupacional en el que se desempeñarían los egresados, de acuerdo con las formaciones profesionales específicas recibidas.

En cuanto a las características de las carreras pensadas para los institutos, Herrera proponía una duración que oscilaba entre los 2 y 3 años. El objetivo era lograr un aumento en el número de egresados. En esta misma línea, proponía equivalencias para aquellos estudiantes que ya hubieran realizado estudios técnicos previos, como así también para aquellos que hubieran realizado trabajos que requirieran de determinadas competencias técnicas. En este último caso, los estudiantes deberían realizar una demostración práctica de sus habilidades.

\section{Oferta curricular y plan de estudios}

La estructura del plan de estudios estaría organizada en tres etapas, una primera de formación básica, en donde los alumnos asistirían a materias y 
cursos que contemplasen aspectos relativos a la comunicación, la cultura en general (humanística y social) y el interés de personal, así como materias o cursos fundamentales de ciencias básicas relacionadas con la especialidad elegida. La segunda etapa sería la de formación técnica, en donde los alumnos adquirirían habilidades técnicas (como resistencia de materiales, termodinámica, mecánica de fluidos, etc.) y técnicas especializadas. La última etapa propuesta es la de práctica profesional, la cual adquiriría diversas formas de acuerdo a la especialidad, pero básicamente implicaría un contacto con el mundo del trabajo y experiencias progresivas que culminasen con un último período dirigido. Al finalizar, los alumnos rendirían un examen global por medio del cual deberían demostrar el logro de los objetivos básicos de las carreras. En caso de que ello no ocurriera, se establecerían mecanismos de recuperación que satisficieran las condiciones curriculares previstas.

En relación a qué carreras se deberían dictar en los IPES, Herrera expresaba la necesidad de definirlo en base a la realidad de la región, lo que indicaría a su vez la localización de los institutos. Estos criterios deberían evaluarse en forma conjunta para tomar las decisiones sobre las especialidades a adoptar. En todos los casos, afirmaba, era preferible establecer carreras que permitieran un campo relativamente amplio de actividad. Al diseñar el currículo, asimismo, se debería establecer el alcance nacional de los títulos, posibilitando la migración de los egresados y la habilitación profesional en todo el país, sin importar el lugar donde fue obtenido el título. En este sentido, el rector sostenía que la nación y las provincias -en ejercicio del poder de policía en sus respectivas jurisdicciones- deberían dictar normas reglamentarias a los fines de garantizar la actividad profesional de los egresados de estos Institutos Superiores, asegurándoles el eficaz desarrollo de su actividad e impidiendo que la realicen quienes no ostenten la idoneidad técnica que comprende la habilitación de su título.

El proyecto tuvo una recepción favorable por parte de los rectores presentes en la reunión. El ingeniero Zapiola, rector de la Universidad Nacional del Comahue, sostuvo que se llevó a cabo una experiencia similar en su región con los Centros de Transferencia y Capacitación. Dada la importancia del proyecto, los presentes decidieron poner el tema en el orden del día de la reunión del mes siguiente ${ }^{35}$. Sin embargo, no se lo volvió a tratar. Por el contrario, las peticiones para la creación de nuevas universidades nacionales en diferentes provincias con sus respectivos proyectos de factibilidad conti-

${ }^{35}$ Consejo de Rectores de Universidades Nacionales, Actas del Consejo de Rectores de Universidades Nacionales (Buenos Aires, 1972). 
nuaron llegando y el CRUN mantuvo sus discusiones mensuales en torno a esta nueva realidad nacional. La creación de nuevas universidades nacionales continuó hasta 1975, con un total de 16 nuevas instituciones distribuidas por todo el territorio ${ }^{36}$.

\section{Similitudes con modelos extranjeros}

El proyecto de Herrera debe enmarcarse en la problemática general que atravesaba la educación superior en la Argentina como consecuencia, principalmente, del aumento de la matrícula desde mediados del siglo XX. Pero también debemos analizarlo en el marco de las transformaciones que atravesó todo el sistema de educación postsecundaria en el mundo en el período de posguerra. Tal como expresamos en secciones anteriores, si bien en un primer momento se vinculó la expansión universitaria con el desarrollo económico, al poco tiempo también comenzó a vincularse con los proyectos de democracia en cada espacio nacional. En Latinoamérica, el principal impulso estuvo vinculado con las ideas de desarrollo económico, promovidas principalmente por organismos internacionales. La idea de que la ciencia y la técnica permitirían acelerar los procesos de industrialización estuvieron a la orden del día y la educación pasó a ser considerada una inversión y ya no un gasto. Así se fueron delineando los proyectos educativos en la Argentina desde principios de la década de 1960 en adelante.

Lo que no se puso en discusión en aquellos años, sea por la impronta de los organismos internacionales o por la coyuntura política que atravesó el país, fue el acceso de nuevos sectores sociales a la educación superior. Dicha cuestión sí formó parte de los principales debates en otros países, como es el caso de EE. UU. El acceso masivo obligó a redireccionar las políticas de educación postsecundaria en distintas partes del mundo y con ello, tal como ya hemos mencionado, se llevó a cabo un proceso de diversificación institucional y diferenciación en los trayectos de formación profesional.

En EE. UU., Alemania y Francia, como hemos visto, se establecieron trayectos de formación diferenciados. Los Community Colleges, son tal vez el ejemplo más claro y con una diferenciación explícita desde sus inicios. Estas

${ }^{36}$ Para un análisis más detallado del proceso de creación de nuevas universidades nacionales durante el período 1971-1975, véase Mariana Mendonça, "La primera ola de expansión universitaria en la Argentina: consecuencias en el mediano plazo", Anuario de la Educación, v. 19 (2018) 
instituciones surgieron a mediados de 1920 en Illinois cuando los colegios comenzaron a ofrecer cursos de los primeros dos años de educación postsecundaria. Unos años después, sucedió algo similar en California, cuando los colegios empezaron a ofrecer los primeros dos años de la universidad. Durante la década del veinte, muchos otros distritos copiaron la iniciativa y al poco tiempo se separaron de la escuela secundaria y se conformaron los $\mathrm{Ju}$ nior Colleges. Al poco tiempo el nombre cambió a Community Colleges, con el objetivo de quitarle la connotación negativa de "Junior" y, a su vez, establecer un vínculo directo con las comunidades. Luego de la segunda guerra mundial, se implementaron una serie de políticas sociales para aquellos soldados que quisieran continuar sus estudios postsecundarios, situación que duplicó la matrícula en los Community Colleges, entre 1944 y $1947^{37}$.

Los estudiantes que asisten a éstas instituciones tienen un perfil muy distinto de aquellos de las universidades con programas de 4 años de duración. Muchos de éstos estudiantes tienen un promedio de 30 años, trabajan a tiempo completo y son el sostén económico de sus familias. Consecuentemente, la oferta académica, así como las distintas instancias de cursada se han adaptado a ello. Muchos cursos se ofrecen los fines de semana y se han hecho enormes esfuerzos para poder ofrecerlos en sedes cercanas a los estudiantes. La oferta académica, por otra parte, ofrece clases teóricas o académicas y fuertemente orientadas en las "ocupaciones del mundo real". Así, el objetivo es encontrar una sinergia entre lo académico y lo vocacional. Otra particularidad es que ofrecen las instalaciones y la infraestructura para que empresas brinden cursos de formación. Destaca, asimismo, el hecho de que la mayoría son de gestión pública, situación que se ve reflejada en el rol que juegan estas instituciones, la población que asiste a ella, y los fondos que recibe de los gobiernos locales con el objetivo de promocionar la igualdad de oportunidades ${ }^{38}$.

A partir de esta breve descripción es posible encontrar puntos en común con la propuesta de Herrera en 1972. Entre ellos, cabe destacar la necesidad de dar respuesta a la masificación de la matrícula, brindando igualdad de oportunidades, así como también la intención de orientar la formación postsecundaria hacia carreras técnicas, con una rápida inserción laboral y directamente vinculada con el medio local. El proyecto de Herrera, en defini-

${ }^{37}$ Claudio de Moura Castro, Andres Bernasconi, y Aimee Verdisco, Community Colleges: Is There a Lesson in Them for Latin America? (Sustainable Development Department Technical Papers Series, 2001).

${ }^{38}$ De Moura Castro et. al. Community Colleges. 
tiva, procuraba diferenciar los trayectos de formación profesional sin obturar la posibilidad de continuar estudios universitarios una vez culminado el primer tramo de formación técnica. Con ello, se intentaría disminuir la tasa de abandono, aumentar la tasa de graduación y a su vez orientar la formación hacia carreras de interés nacional, regional y/o local.

A diferencia de lo ocurrido en otros países, sin embargo, en la Argentina no se llevaron a cabo propuestas similares a aquellos, sino que se dio inicio a una expansión de universidades públicas a lo largo y ancho de todo el país. Los problemas de la alta tasa de matrícula, baja tasa de graduación, alta tasa de abandono, la extensión en el tiempo de cursada, entre otros problemas que presentaba el sistema de educación superior en este país no fueron resueltos. Por el contrario, volverían a estar en la agenda en la década del noventa cuando se llevó a cabo la segunda ola de expansión, y siguen siendo temas de discusión de las políticas educativas en el siglo XXI. De acuerdo a Krotch, la imposibilidad en el avance de una política de aquellas características debe ser analizada en el marco del sistema productivo de la Argentina. En este sentido, sostiene que el nuevo perfil en la formación de los estudiantes de educación superior no puede derivarse únicamente de políticas públicas tendientes a resolver los problemas de la congestión de las universidades, sino que debe surgir a partir de las demandas concretas de la economía ${ }^{39}$.

Desde nuestro punto de vista, entendemos que, si bien no se ha logrado plasmar una alternativa diferenciada en los trayectos de formación de manera explícita, la misma se ha desarrollado a lo largo de los años de manera implícita. La alta tasa de abandono en los primeros años de la formación universitaria, implícitamente, ha dado forma a trayectos de formación diferenciados. Tanto es así que, a partir de la década de 1990 ha ganado cada vez más fuerza la práctica de ofrecer títulos intermedios, en los que se acreditan los conocimientos adquiridos por los estudiantes en los primeros años de formación.

\section{A modo de cierre}

Hacia mediados del siglo XX hubo una fuerte transformación en el sistema de educación superior a nivel mundial. El aumento de la matrícula obligó a repensar las formas y el contenido de la formación postsecundaria en distintas partes del mundo y, consecuentemente, se llevó a cabo un proceso de

\footnotetext{
${ }^{39}$ Krotch, Educación Superior:169.
} 
diversificación y diferenciación en los trayectos de formación profesional. La Argentina no estuvo exenta, pero a diferencia de muchos otros países, no hubo una diferenciación explícita. La oferta se diversificó con instituciones públicas y privadas, universitarias y no universitarias, pero todas ellas con trayectos de formación de carreras de 4 años como mínimo. Sólo en contados casos se ofrecieron títulos de tres años de formación, y recién hacia la década de 1990 comenzaron a tener mayor peso.

La política que predominó, en cambio, fue la expansión de instituciones universitarias. En el sector público, tras la creación de 2 universidades nacionales en 1956, la expansión continuó con una nueva universidad en 1969, mientras que entre 1971 y 1975 se sumaron 16 nuevas casas de estudio a lo largo y ancho del país. Si bien la propuesta consistía en crear nuevas universidades nacionales con una fuerte distinción respecto de las ya existentes, había otros problemas a resolver en el sistema de educación superior. La alta tasa de la matrícula, la alta tasa de abandono, y la baja tasa de graduación fueron los ejes de mayor preocupación entre los referentes de educación. Consecuentemente, así como en otros países se procuró establecer trayectos de formación diferenciadas, con titulaciones de 1 o 2 años, con posibilidad luego de extender y completar un trayecto de 4 años, aquí en la Argentina se propusieron alternativas similares, a los fines de evitar la reproducción de las falencias propias que exhibían las universidades nacionales. Los IPES fueron una de las tantas opciones discutidas en los años aquí estudiados, con una fuerte impronta en trayectos de formación diferenciados, sin por ello eliminar la posibilidad de una formación universitaria.

Sin embargo, pese a haber tenido una muy buena recepción por parte de los rectores, dicha propuesta quedó anulada tras el vertiginoso proceso de expansión institucional que tuvo lugar entre 1971 y 1975 . El resultado final, lejos de avanzar en alternativas para mejorar la performance de las universidades, con una mejora en las tasas de graduación y una disminución en las tasas de abandono, fue la reproducción de los problemas que habían sido diagnosticados. Las tasas de abandono en los primeros años se mantuvieron en niveles altos, situación que resulta, de hecho, en trayectorias de formación diferenciadas, con la particularidad de que los estudiantes no obtienen un título que acredite sus años de formación. Recién en la década de 1990 y con mayor fuerza en la última ola de expansión universitaria iniciada en 2008, comenzaron a ofrecerse titulaciones intermedias en las universidades nacionales. Cabe preguntarse, entonces, por qué la expansión institucional tomó ésta última forma y no una similar a aquella que experimentaron otros países. 
Pese a que no se llevó a cabo una diferenciación explícita en los trayectos de formación, las altas tasas de matriculación y las altas tasas de abandono en los primeros años de la cursada, dan cuenta de trayectos de formación diferenciados. El ingreso al mundo del trabajo con una carrera universitaria incompleta, condición en la que se halla un gran número de trabajadores con estudios superiores en la Argentina desde hace décadas, ha buscado ser subsanado mediante la oferta de tecnicaturas, o titulaciones intermedias desde mediados del siglo XX en adelante. Sin embargo, la expansión de instituciones universitarias públicas, con trayectos de formación de 5 años, han liderado las políticas de educación superior desde 1970 hasta la actualidad. Recién en las últimas décadas han comenzado a ofrecerse títulos intermedios que permiten acreditar la formación de los primeros años de la educación superior.

Queda pendiente para futuros trabajos, entonces, avanzar en el conocimiento de las características de éstos nuevos trayectos de formación intermedios y el rol que juegan en la formación de la fuerza de trabajo.

\section{Bibliografía}

Altbach, Phillip, Reisberg, Liz, \& De Wit, Hans. Responding to massification. Differentiation in Postesceondary Education Worldwide. Rotterdam: Sense Publishers and Körber Foundation, 2017.

CANo, Diego. La educación superior en la Argentina. Buenos Aires: Grupo editor latinoamericano, 1985.

ChIRoleu, AdRiana. "La modernización universitaria en la agenda de gobierno argentino: lecciones de la experiencia”. En. Fundamentos en humanidades, v. V, n. 9 (2004): 29-44.

Chiroleu, Adriana, Suasnádar, Claudio \& Rovelli, laura. Política universitaria en la Argentina: revisando viejos legados en busca de nuevos horizontes. Los Polvorines: Universidad Nacional de General Sarmiento, IEC-CONADU, 2012.

De Moura Castro, Claudio., Bernasconi, A.ndrés \& Verdisco, Aimee Community Colleges: Is there a lesson in them for Latin America? Washington, D.C.: Education Unit. Inter-American Development Bank, 2001.

Del Bello, Juan Carlos., Barsky, Osvaldo \& Giménez, Graciela. La universidad privada argentina. Buenos Aires: El Zorzal, 2007.

Ezcurra, ANA María. Igualdad en educación superior. Un desafío mundial. Los Polvorines: IEC-UNGS, 2011. 
Garcías De Fanelli, ANa María \& Balán, Jorge. Expansión de la oferta universitaria: nuevas instituciones, nuevos. Buenos Aires: CEDES, 1994.

HERRERA, JULIO. Una política de expansión del nivel terciario. Mendoza: Universidad Nacional de Cuyo, 1972.

Holm-Nielsen, Lauritz. B., Thorn, Kristian, Brunner, JoAquin \& Balán, JORGE. "Regional and international challenges to higher education in Latin America". En Higher Education in Latin America. The international dimension, editado por Hans de Wit, Isabel Cristina Jaramillo, Jocelyne Gacel-Ávila, \& Jane Knight. Washington D. C.: The International Bank for Reconstruction and Development/The World Bank, 2005.

Jung, MARÍA EugENIA." Derechas y universidad en los sesenta. Lecturas inspiradoras y modelos universitarios: Tres estudios de caso en Uruguay y Argentina". En Cuadernos de Marte, V(17), (2019): 151-181.

KROTSCH, PEDRO. Educación superior y reformas comparadas. Bernal: Universidad Nacional de Quilmes, 2009.

MENDONÇA, MARIANA. "Los (frustrados) intentos de modernización en la Universidad de Buenos Aires desde su creación hasta el rectorado de Devoto (1821-1968)". En Universidad, política y movimiento estudiantil en la Argentina entre la "Revolución Libertadora" y la democracia del 83", editado por Mariano Millán. Buenos Aires: Final Abierto, 2014.

MendonçA, Mariana. "Cómo resolver el problema universitario: nuevos diagnósticos y cambios en la agenda política durante el onganiato (1966-1970)". En Historia da educaçao, v. 19, n. 47 (2015): 229-248.

MEndonÇA, MARIANA. "Nuevas universidades en la década del setenta. Apuntes para un análisis crítico del proceso de expansión del sistema de educación superior en la Argentina". En PolHis Revista Bibliográfica del Programa Interuniversitario de Historia Política, v. 18, n. 9 (2017): 286.

MENDONÇA, MARIANA. "Creación, nacionalización y escisión: ¿reestructuración? Una aproximación al proceso de transformación del sistema universitario argentino (1971-1973)". En Revista Iberoamericana de Educación Superior, v.9, n.24 (2018): 82-105.

MEndonçA, MaRiana. "La primera ola de expansión universitaria en la Argentina: consecuencias en el mediano plazo". En Anuario Historia de la Educación, v. 19 (2018):24-49.

RAMA, CARLOS. La tercera reforma de la educación superior en América Latina. Buenos Aires: Fondo de Cultura Económica, 2006.

RovelLI, LAURA. "Del plan a la política de creación de nuevas universidades nacionales en Argentina: la expansión institucional de los años 70 “. En Temas y Debates , v. 17 (2009): 117-137. 
Tavela, Danya \& Catino, Magalí. Áreas de vacancia. Vinculación, pertinencia del sistema universitario. Buenos Aires: Secretaría de Políticas Universitarias-Ministerio de Educación de la Nación, 2018.

Trow, MARTin. Problemas in the transition frome elite to mass higher education. Berkley: Institute of International Studies, University of California, 1973. 